\title{
Desplazamiento respecto de los saberes disciplinares de la Fonoaudiología
}

\author{
Displacements with respect to disciplinary \\ knowledge of Speech, Language and Voice Therapist
}

\section{Luis Romero R. \\ Académico \\ Profesor Asociado \\ Escuela de Fonoaudiología \\ Universidad de Chile}

\section{RESUMEN}

Este artículo presenta una serie de propuestas acerca de lo que implica ser fonoaudiólogo/a. Se contextualiza en el marco del proceso de la innovación curricular propuesta por la Universidad de Chile para todas las Carreras, en particular las de la salud y entre ellas Fonoaudiología. Luego de esta contextualización, la idea es presentar un eje de análisis del (posible) saber disciplinar y los supuestos que están en la base de la formación de este profesional. Para ello se realiza un recuento histórico de la profesión. Se consideran las modificaciones contextuales y epistemológicas que sostendrían el saber disciplinar actual y que tendrían relación con el bienestar comunicativo de las personas. Cómo tal éste es un ejercicio inédito en Chile. En él, las personas se enfrentan al dilema de establecer cuál es el saber disciplinar de la profesión de fonoaudiólogo/a, de proponer la episteme de la fonoaudiología. Este enfoque cualitativo de final abierto, no se ha utilizado en estudios anteriores.

Palabras claves: saber disciplinar, fonoaudiología.

\section{ABSTRACT}

This article presents a series of proposals about what it actually means to be a speech pathologist. The theoretical framework that underlies these proposals is based on the curricular innovation process that the careers at the University of Chile are undergoing, especially for those in the health area, including Speech Therapy. The analysis is done on the basis of two main issues: a definition of disciplinary knowledge and the assumptions that underlie the training of professionals pursuing the degree of speech therapists. The article provides a historical account of the development of speech therapy, including the contextual and epistemological changes that may have given rise to the current status and knowledge of this discipline, which are related to the communicative welfare of people. As such, this paper provides new insights into the understanding of the relationships that this discipline may establish in the Chilean context. Thus, professionals are faced up with the dilemma of defining what the key tenets of the disciplinary knowledge of the speech-language pathologist are, and to propose the episteme of speech therapy. This qualitative open ended approach has not been used in previous studies.

Key words: disciplinary knowledge, speech-language pathologist. 


\section{Introducción}

Este artículo representa una reflexión acerca de lo que implica ser fonoaudiólogo/a, su saber disciplinar y los supuestos que están en la base de su formación. Dicha reflexión ha resultado un ejercicio clave en el contexto de las innovaciones curriculares propuestas para la Escuela de Fonoaudiología, Facultad de Medicina de la Universidad de Chile.

Este texto es también una narración de los eventos. Pretende dejar de manifiesto el intento por caracterizar los fenómenos de la propia experiencia reflexiva. La narración se hace guiada por una noción de temporalidad, vinculando afectos e incertidumbres, conjunto que permite una construcción que se espera, sea coherente y con sentido.

En este afán, a través de este texto se abordarán consideraciones con respecto al ámbito fonoaudiológico relacionados con su historia en Chile; la relación de la fonoaudiología con la epistemología en la construcción de "conocimiento cierto" de índole científico y humanista; y finalmente el intento por aproximarse a nociones fundamentales que permiten caracterizar el quehacer del/de la fonoaudiólogo/a y que dan luces, por lo tanto, sobre el establecimiento de los saberes que resaltan a esta disciplina fonoaudiológica de otras disciplinas vinculadas a la salud y a la comunicación de los seres huma (vinculada a la salud y la educación).

Es importante señalar que este ejercicio es inédito en Chile. En él, se enfrenta el dilema de establecer cuál es el saber disciplinar de la profesión de fonoaudiólogo/a, de saber cuál es la episteme de la fonoaudiología. Esto ocurre de una forma tal que los sujetos implicados comienzan a elaborar y a pensar en sí mismos como objetos de análisis y reflexión: los/las fonoaudiólogos/as pensando en ellos, en su hacer, su disciplina y qué es lo que la hace propia y característica y, por lo tanto, diferenciable de otras disciplinas profesionales del área de la salud y de otras entidades académicas de Educación Superior.

\section{Necesidad de la creación de la Carrera de Fonoaudiología desde una perspectiva histórica}

Históricamente la fonoaudiología nace como carrera en la Universidad de Chile en el año 1970. Lo hace bajo el alero de la Facultad de Medicina de la Universidad de Chile, con una fuerte orientación técnica y pedagógica, en donde la visión del currículo fue de logros de objetivos y de colaboración con el quehacer médico.

Poco a poco, en un proceso de evolución de la Carrera, se asentaron las bases sobre las cuales se intentó responder a la pregunta auténtica acerca de cuál sería el saber disciplinar de la fonoaudiología. Fue en 1999 que se explicitó formalmente la primera declaración que intentó establecer los saberes disciplinares de la fonoaudiología. En ese entonces se la consideró como:

"la disciplina cuyo objeto de estudio es la comunicación humana oral como elemento principal de interacción entre los individuos, sus 
trastornos y las estrategias diagnósticas y terapéuticas que hacen posible su recuperación"1 ${ }^{1}$.

Esta declaración se gestó en la Carrera de Fonoaudiología. En ella se puede constatar la influencia del modelo médico, pero además integra aspectos de índole social y humanista.

En la actualidad, la Carrera de Fonoaudiología está inserta en el proceso de innovación curricular que la Universidad de Chile puso en marcha desde el año 2007.

Respondiendo al proceso de cambio, las personas que conforman el claustro de la Escuela de Fonoaudiología de la Universidad de Chile, se propusieron re - pensar el hacer, la disciplina, el saber científico y no científico, los métodos de validación del conocimiento de éste, etc. En síntesis la episteme de fonoaudiología.

\section{Reflexiones desde la epistemología}

Al indagar desde una perspectiva epistemológica cualquier situación general, se tratar de entender el problema fundamental de la epistemología: la relación sujeto-objeto. Al hacerlo en particular en al ámbito de la fonoaudiología, pareciera que se está cumpliendo con la acción cognitiva fundamental para sintonizar a los actores en la tarea de abocarse a la comprensión de quiénes son los sujetos en sí mismos en tanto sujetos que conocen. Esto incluye cualquiera otra situación que se reflexione como posible y que se relacione con el/la fonoaudiólogo/a de la Universidad de Chile. De acuerdo a esta teoría, se le llama "sujeto" al ser cognoscente y "objeto" a todo proceso o fenómeno sobre el cual el sujeto desarrolla su actividad cognitiva. De este modo, el problema se presenta en la relación de quien conoce y lo que es cognoscible.

La intención es explicar desde un punto de vista de la fonoaudiología como disciplina, cuál es la relación sujeto - objeto, en el contexto de un sujeto cognoscente (fonoaudiólogo/a) y su objeto (en este caso, su saber disciplinar). Tener éxito en esta tarea implica reconocer el saber que representa la disciplina fonoaudiológica.

Se propone un método para ello, el cual por supuesto no está pre - escrito. Consiste en pensar que la epistemología en realidad no es una ciencia inventiva, es decir, que no pretende hacer el gran descubrimiento acerca del hecho del conocimiento humano, slno más bien explicativa de un hecho que el ser humano ya es poseedor: el hecho de conocer ${ }^{2}$. Lo que se hace en realidad es acceder a este hecho a través de la reflexión, dispositivo humano que permite hacer la introspección necesaria para poder asumir que la conciencia se mira a sí misma, vuelta sobre sí misma. En resumen, este método implica una focalización sobre los elementos que constituyen "ser fonoaudiólogo/a", su saber disciplinar y los supuestos que estarían en la base de la formación de un/una fonoaudiólogo/a de la Universidad de Chile. 


\section{Mirada actualizada a la luz de los cambios del hacer disciplinar y los saberes que se identifican como propios.}

Teniendo presente lo dicho en los párrafos anteriores, se observa un desplazamiento de racionalidades, desde una perspectiva que considera la fonoaudiología como "Ia disciplina cuyo objeto de estudio es la comunicación humana oral como elemento principal de interacción entre los individuos, sus trastornos y las estrategias diagnósticas y terapéuticas que hacen posible su recuperación" ${ }^{1}$, a pensarla como la disciplina que se ocupa del "bienestar comunicativo de las personas". Al hacer esto se asume como supuestos que la comunicación es un fenómeno amplio, complejo y que puede ser mirado desde muchas perspectivas al igual que la noción de bienestar. En la caracterización de esta episteme están descritos procesos absolutamente relacionados con la primera declaración de 1999. Pero hay más. Para ello es necesario hacer algunas precisiones semánticas. En primer término con relación a la noción de "bienestar".

Según el diccionario de la RAE, "bienestar" presenta varia acepciones: 1 . Conjunto de las cosas necesarias para vivir bien. 2. Vida holgada o abastecida de cuanto conduce a pasarlo bien y con tranquilidad. 3. Estado de la persona en el que se le hace sensible el buen funcionamiento de su actividad somática y psíquica.

Por otra parte, la noción de bienestar que se tomará como referencia para este trabajo, está referida a las actitudes y comportamientos que mejoran la calidad de vida y ayudan a llegar a un estado de salud óptima. Se trata entonces de un proceso activo por parte del sujeto dirigido a mejorar su propio estilo de vida en todas sus dimensiones ${ }^{3}$. Representa las acciones que permiten el desarrollo de un nivel adecuado de salud general. El bienestar deseado, entonces, se obtiene mediante hábitos que generan $o$ se desplazan hacia una adecuada adaptación e integración de las dimensiones físicas, mental, social, espiritual y emocional a cualquier nivel de necesidad de salud de un sujeto cualquiera. Esto implica que podemos experimentar bienestar, ya sea que se esté en enfermedad o salud. Implica también que es posible encontrar sujetos con diversidad de necesidades de salud. En torno a esta noción de bienestar es en la que el/la fonoaudiólogo/a debiera actuar. $\mathrm{Y}$ debiera hacerlo en concordancia con esa diversidad de necesidades desde el punto de vista de su quehacer. Se reflexiona, por lo tanto, en torno a esta noción de bienestar y de cómo el/la fonoaudiólogo/a podría contribuir a la condición de las personas por medio de este saber.

Es cierto que esta noción de bienestar puede aun resultar muy amplia y no totalmente orientadora respecto de lo que debiera caracterizar el saber y el quehacer del/de la fonoaudiólogo/a. Una forma de hacer coherente esta idea con la acción de este/esta profesional es entender que en el ámbito de la diversidad de necesidades de bienestar de cualquier sujeto, existen muchas en relación a la comunicación. Es decir, es posible entender a las personas con diversidad de necesidades de bienestar relacionadas con la comunicación. El/la fonoaudiólogo/a estaría 
construido para acompañar a los sujetos e intentar satisfacer o ayudar a satisfacer las diversas necesidades de bienestar comunicativo que los sujetos puedan presentar.

La comunicación humana, por lo tanto, es otro de los aspectos a abordar y tratar de integrar y asociarlo con la noción de bienestar antes planteado.

Según la RAE, "comunicación" deriva del latín communication, -ōnis. $\mathrm{Y}$ se relaciona con la "acción y efecto de comunicar o comunicarse". Con la “transmisión de señales mediante un código común al emisor y al receptor".

En otros textos se puede encontrar referencias desde el punto de vista de la Teoría de la Comunicación:

Desde el primer tercio del siglo XX hasta la actualidad, la teoría de la comunicación se ha ido construyendo desde perspectivas muy diferentes. Desde la teoría físico-matemática de Shannon y Weaver, conocida como "Teoría matemática de la información", hasta la teoría psicológica con base a la percepción propuesta por Abraham Moles, pasando por una teoría social con base en la lengua -Saussure-, con base en la antropología cognitiva -Lévi Strausso con base en los enfoques interaccionistas y sistémicos -Bateson, Watzlawick, Goffman. Y más aún, también han destacado las aportaciones en el campo de los efectos de la comunicación de masas, un ámbito representado por nombres como Lasswell, Lazarsfeld, Berelson y Hovland, y las teorías críticas de la comunicación, promovidas desde la Escuela de Frankfurt por intelectuales como Adorno, Horkheimer y Marcuse, entre otros. Este panorama pone en evidencia la complejidad del asunto, las múltiples aportaciones con que se ha tratado de dotar de coherencia a lo que conocemos como Teoría de la Comunicación. Ello es resultado, entre otros factores, de la polisemia misma del concepto de comunicación ${ }^{4}$.

De acuerdo a la teoría de la comunicación, ella misma se define como un "conjunto de elementos en interacción en donde toda modificación de uno de ellos afecta las relaciones entre los otros elementos" ${ }^{5}$.Es por estas condiciones (entre muchas otras) que se le ha llamado teoría sistémica de la comunicación. En ésta se significa con mayor o menor claridad lo que se entiende por interacción comunicativa, dando a entender la importancia que tiene en las relaciones humanas.

Es significativa también la Teoría Crítica de la Comunicación, la cual se ha abierto pensar la comunicación como un fenómeno social, vista como útil para la transformación de los espacios colectivos. Debe servir además:

\footnotetext{
“como plataforma para el desarrollo de estrategias discursivas, propuestas técnicas o espacios de mediación capaces de ahondar en una radicalización de la democracia, la solidaridad y el conocimiento-emancipación, como puntales de la renovación del paradigma epistemológico dominante a partir de estos momentos de crisis postmoderna" ${ }^{6}$.
} 
Más allá de cualquier enfoque o teoría de la comunicación desarrollada o por construir, se requiere declarar en este punto que el/la fonoaudiólogo/a que sea capaz de concebirse, pueda contar con las competencias para construir relaciones de inter - subjetividad, apoyado en el uso de su discurso, lenguaje, habla, voz, audición, o en ausencia de alguna de ellas, considerando los aspectos pragmáticos y en general de uso del lenguaje en función de una comunicación siempre adecuable. De tal manera que este profesional, sustente la comunicación desde una perspectiva saludable, intentando aportar con el bienestar comunicativo de un "otro" que lo requiere.

\section{Aproximación a los saberes fundantes de la fonoaudiología (en Chile) o las nociones de bienestar comunicativo de las personas.}

En esta mirada es posible señalar la convergencia de las nociones de "bienestar" y “comunicación" dialogando entre ellas. Es factible reflexionar y actuar en torno de la condición y calidad de vida de las personas y su desempeño comunicativo, en cuanto a seres sociales con identidad. En otras palabras, se puede pensar y concebir al/a la fonoaudiólogo/a sabiendo actuar con el propósito de contribuir al bienestar comunicativo de las personas. Un/una fonoaudiólogo/a que posee las competencias para responder y crear nuevas respuestas en torno a las necesidades comunicativas que los sujetos manifiesten en las más diversas condiciones sociales y culturales. De estas afirmaciones surgen algunas preguntas, mismas que orientan el desarrollo reflexivo: ¿Qué tiene qué saber un sujeto para poder llegar a incidir en el bienestar comunicativo de las personas? O dicho de otra forma ¿De qué manera el/la sujeto fonoaudiólogo/a puede llegar a constituirse como tal?.

Estas preguntas mueven a pensar necesariamente en el proceso formativo de los/las fonoaudiólogas/os. Y es precisamente en torno a este tema que se propone la formación de un/una fonoaudióloga/o a través del tránsito por diversos dominios (ámbitos de acción). Estos permitirían al estudiante de fonoaudiología desarrollar una racionalidad fonoaudiológica, es decir, una manera de enfrentar y resolver los problemas complejos desde una perspectiva característica, propia. Los desempeños que caracterizan al/la fonoaudiólogo/a dan cuenta de esta racionalidad o manera de pensar. En general tiene que ver con anticiparse a los hechos, prever escenarios, abordar problemas, evaluar efectos e impactos de situaciones complejas, hacerse responsable de las consecuencias de sus actos, sabiendo además, actuar de manera óptima en medio de la incertidumbre.

En este caso, todas estas actuaciones estarían vinculadas con el acompañamiento a sujetos para que estos logren bienestar comunicativo. Para ello es necesario proponer que la acción del/de la fonoaudiólogo/a está guiada por una forma de pensamiento más bien ecléctica ${ }^{1}$.

\footnotetext{
1 Desde una óptica filosófica es considerado el eclecticismo como el intento infructuoso de conciliar elementos excluyentes entre sí, por ser incapaz de delimitar sus nexos esenciales en relación con la situación en que se aplica, de tomar de cualquier parte por conveniencia y elegir categorías muy diversas ${ }^{7}$. Es decir, aquella mezcla que no obedece a principios determinados, distintos y hasta contrapuestos. Su principal defecto metodológico radica en su incapacidad para delimitar, en la suma de nexos y relaciones los lazos fundamentales del objeto, del fenómeno respecto al medio que lo circunda, lo cual le impide hallar el eslabón principal en la cadena de acontecimientos ${ }^{7}$.
} 
Es decir, un actuar caracterizado por combinar, asociar y mezclar coherentemente puntos de vista, ideas, valoraciones y estrategias de acción, de acuerdo a las necesidades comunicativas de la persona a la que se acompaña ${ }^{7}$.

En la formación del/de la fonoaudiólogo/a uno de los dominios clave es el de intervención. Este dominio se entiende como el ámbito de acción que implica relacionarse con personas que tengan necesidades de ayuda fonoaudiológica en cualquiera etapa del ciclo vital. Implica también, que la formación en este dominio involucra el desarrollo de las competencias que habilitan a los egresados para construir espacios de intervención evaluando, diagnosticando, tratando y potenciando la comunicación y motricidad orofacial, mediante el abordaje de las áreas de lenguaje, habla, voz, audición y funciones orofaciales.

A través de la formación en este dominio (conjuntamente con otros), comienza a construirse la "racionalidad fonoaudiológica", la cual lejos de establecerse como un elemento fijo y estático, es un proceso que se desplaza y se va enriqueciendo en la medida del tránsito del estudiante a través de la Carrera --en primer término-- y luego en la experiencia laboral al acompañar a sujetos que requieren satisfacer necesidades comunicativas de distinto orden. En este artículo se entiende como racionalidad fonoaudiológica a una manera de enfrentar y resolver los problemas o familia de problemas, resguardando, obviamente, las capacidades de cada sujeto fonoaudióloga/o. Los desempeños que caracterizan al/a la fonoaudiólogo/a debieran dar cuenta de esta racionalidad o manera de pensar. La racionalidad profesional en general (y también la fonoaudiológica) requiere actuar terapéuticamente de manera integral en conjunto con otros actores relevantes, considerar el contexto, la diversidad y los derechos de las personas y respetando las normas éticas ${ }^{8}$.

Se propone, además, que el estudiante de fonoaudiología transite por otros dominios. Al declarar estos como instancias de formación se está abordando la diversidad que constituye al propio estudiante y que mínimamente debiera considerar en cuanto a saber disciplinar que caracteriza la formación del/de la fonoaudiólogo/a. Así, surge el dominio de la formación común, el de investigación o científico, de promoción y prevención. Fue parte del trabajo de la comunidad de la Escuela de Fonoaudiología de la Universidad de Chile, la descripción detallada y pormenorizada de lo que cada uno de los dominios implica en términos de la formación de cada uno de las/los fonoaudiólogas/os.

\section{Comentarios}

El presente documento es una reflexión acerca del saber disciplinar de la Fonoaudiología en Chile. Establecer estos saberes redunda en la disciplina misma y se refleja en las condiciones que determinan la experticia del/de la fonoaudiólogo/a y los supuestos que están en la base de su formación. Dicha reflexión se presenta en el marco de una importante innovación curricular. En ella se hace la pregunta auténtica acerca de cuáles son los saberes disciplinares de la fonoaudiología. 
Intentando llegar a buen puerto se hace una contextualización histórica (quiénes éramos), luego se proyecta el perfil al presente con debilidades y fortalezas (quiénes somos). Esto nos permite pensar en que somos sujetos profesionales en términos del bienestar comunicativo nuestro y de los demás, constantemente siendo, en evolución y cambio debido a lo complejo de este saber disciplinar (quiénes podremos llegar a ser).

La matriz de este escrito no es del todo individual. Está influenciada por una reflexión colectiva de la comunidad Escuela de Fonoaudiología de la Universidad de Chile y como tal ha resultado un ejercicio clave en el contexto de la evaluación y propuestas que tienen como propósito satisfacer las necesidades de modernización del pregrado llevadas a cabo a través de la innovación curricular propuesta para esta Escuela.

\section{Referencias}

1. Maggiolo, M; Swalm, E. (1999) Escuela de Fonoaudiología: Notas acerca de su historia. Revista Chilena de Fonoaudiología, 1, (1), p. 6-10.

2. Altisen, Claudio (2001). Epistemología. Visitado en http://www.librosenred.com/libros/epistemologia.html , 20 de mayo 2009.

3. Donatell, Snow \& Wilcox, (1999), p.7, en Laportegui Corsino, Edgar (2001), en: http://www.saludmed.com/Bienestar/Cap1/SalBien.html). visitado 01/06/2009, 9:00 horas.

4. Rizo, M. (2011) El camino hacia la "Nueva Comunicación", Breve apunte sobre las aportaciones de la escuela de Palo Alto". (40). Enhttp://www.razonypalabra.org.mx/anteriores/n40/m rizo.html.

5. Marc, Edmond; Picard, Dominique (1992) La interacción social. Cultura, instituciones y comunicación, Barcelona, Paidós.

6. Teoría Critica de la Comunicación, en COMPOLITICAS. (2011) Grupo interdisciplinario de estudios en comunicación, política y cambio social. Visitado en : http://institucional.us.es/cico/lineas_tcc.htm

7. Ortiz Torres, Emilio, (2000) El Peligro del Eclecticismo en las Investigaciones Psicopedagógicas Contemporáneas. El Caso De Las Concepciones de Vigotsky y Piaget. Revista Pedagogía Universitaria. 5, (3).

http://www.sld.cu/galerias/pdf/sitios/rehabilitaciontemprana/el_peligro_del_eclecticismo_en_las_investig aciones_psicopedagogicas_contemporaneas_1.pdf. Visitado 14/03/2011.

8. Comisión Local de Innovación Curricular de Fonoaudiología (2010) Matriz de intervención. Escuela de Fonoaudiología, Facultad de Medicina. Universidad de Chile. 